\title{
Blood stasis in the descending thoracic aorta and its relationship to cardiac functional parameters
}

\author{
Lauren A Simprini ${ }^{*}$, Gaby Weissman, Anthony R Fuisz \\ From 2011 SCMR/Euro CMR Joint Scientific Sessions \\ Nice, France. 3-6 February 2011
}

\section{Background}

Spin echo Axial T1 images are used to evaluate the great vessels and cardiac structures. In some patients with depressed left ventricular ejection fraction (LVEF) the sequence fails to null blood of the descending aorta (dAo) at the level of the main pulmonary artery (MPA). This may represent stasis of blood from the aorta and reflect a decreased LVEF, stroke volume (LVSV) and/or cardiac output (CO). This finding has not systematically been studied. We evaluated the relationship between opacification of the descending aorta and cardiac functional parameters.

\section{Methods}

148 consecutive cardiac MR scans performed between $7 / 2010$ and 9/2010 that included evaluation of left ventricular function were reviewed. The T1 axial images (SE-EPI TR750TE20Flip90) were evaluated for opacification of some or all of the dAo at the level of the MPA. This was also identified in either the slice above or below this level to be considered a positive finding. The values of LVEF, SV, and CO as determined by manual tracings and application of Simpson's rule were obtained (ViewForum, Philips, Best, The Netherlands).

\section{Results}

Mean LVEF value for patients with no opacification was $60 \%$ while those with any amount of opacification had a mean LVEF of $36 \%(\mathrm{p}<.0001)$. Specificity and sensitivity for LVEF < 55 was $97 \%$ and $32 \%$ respectively, for LVEF < 45 was $95 \%$ and $40 \%$ respectively, and for LVEF $<35$ was $93 \%$ and $53 \%$ respectively. The mean SV value for patients with no opacification was $96 \mathrm{ml}$ while those with any amount of opacification had a mean LVSV of $58 \mathrm{ml}(\mathrm{p}<.0001)$. Specificity and sensitivity for LVSV
Table 1 Specificity and sensitivity of descending aorta opacification

\begin{tabular}{lll}
\hline & Specificity & Sensitivity \\
\hline LVEF $<55$ & $97 \%$ & $32 \%$ \\
LVEF $<45$ & $95 \%$ & $40 \%$ \\
LVEF $<35$ & $93 \%$ & $53 \%$ \\
LVSV $<50 \mathrm{ml}$ & $92 \%$ & $53 \%$ \\
CO $<5$ L/min & $96 \%$ & $38 \%$ \\
\hline
\end{tabular}

$<50 \mathrm{ml}$ was $92 \%$ and $53 \%$ respectively. The mean CO value for patients with no opacification was $6.2 \mathrm{~L} / \mathrm{min}$ while those with any amount of opacification had a mean CO of $4.5 \mathrm{~L} / \mathrm{min}(\mathrm{p}<.0001)$. Specificity and sensitivity for $\mathrm{CO}<5.0 \mathrm{~L} / \mathrm{min}$ was $96 \%$ and $38 \%$ respectively.

\section{Conclusions}

Opacification of the descending aorta at the level of the MPA is a specific but not sensitive finding for depressed LVEF and lower than normal LVSV and CO. When this finding is observed, further evaluation of a patient's ejection fraction should be considered.

Published: 2 February 2011

doi:10.1186/1532-429X-13-S1-P172

Cite this article as: Simprini et al:: Blood stasis in the descending thoracic aorta and its relationship to cardiac functional parameters. Journal of Cardiovascular Magnetic Resonance 2011 13(Suppl 1):P172.

Washington Hospital Center, Washington, DC, USA

(c) 2011 Simprini et al; licensee BioMed Central Ltd. This is an open access article distributed under the terms of the Creative Commons Attribution License (http://creativecommons.org/licenses/by/2.0), which permits unrestricted use, distribution, and reproduction in any medium, provided the original work is properly cited. 